\title{
Running scales in causal dynamical triangulations
}

\author{
Giuseppe Clemente, ${ }^{*}$ Massimo D’Elia, ${ }^{\dagger}$ and Alessandro Ferraro \\ Dipartimento di Fisica dell'Università di Pisa and INFN_Sezione di Pisa, \\ Largo Pontecorvo 3, I-56127 Pisa, Italy
}

(Received 8 March 2019; published 20 June 2019)

\begin{abstract}
The search for typical length scales, eventually diverging at a critical point, is a major goal for lattice approaches looking for a continuum theory of quantum gravity. Within the simplicial Monte Carlo approach known as causal dynamical triangulations, we study the spectrum of the Laplace operator to infer the geometrical properties of triangulations. In some phase of the theory a discrete set of length scales emerges, persisting in the infinite volume limit; such scales run as a function of the bare couplings, consistently with a critical behavior around a possible second order transition.
\end{abstract}

DOI: 10.1103/PhysRevD.99.114506

\section{INTRODUCTION}

The quest for a self-consistent quantum theory of gravity is still far from being settled. Quantum field theory fails in providing solutions which are renormalizable from a perturbative point of view, i.e., with ultraviolet (UV) divergences reabsorbed at all orders in the coupling expansion by adding a finite number of counterterms [1]; however, the possibility of finding a nonperturbative solution has not yet been given up, a promising approach being represented by the so-called asymptotic safety program [2]. The program is rooted in the renormalization group (RG) framework: in a few words, the idea is to find a nonperturbative UV fixed point in parameter space, with a RG-flow line stemming from it and reproducing the theory of gravity at lower energy scales.

Consistent progress in this direction has been achieved by analytic studies of the RG flow [3-6]. A complementary approach is numerical: one considers a discretization of the Euclidean path integral of the theory in configuration space, suitable to be studied by Monte Carlo simulations, and looks for possible critical points, i.e., for values of the bare parameters where the correlation length, measured in units of the elementary discretization scale, diverges. Such points are candidate UV fixed points for a continuum limit of QG. As usual in lattice field theory, one aims to eventually find scaling regions around such points where different physical

\footnotetext{
*giuseppe.clemente@pi.infn.it massimo.delia@unipi.it

\#alessandro.ferraro@pi.infn.it
}

Published by the American Physical Society under the terms of the Creative Commons Attribution 4.0 International license. Further distribution of this work must maintain attribution to the author(s) and the published article's title, journal citation, and DOI. Funded by SCOAP. lengths scale proportionally to each other as a function of the bare parameters: apart from providing evidence of the approach to the continuum, the scaling ratios will contain information relevant to continuum physics (think, e.g., of hadron mass ratios determined by lattice QCD simulations).

A standard discretization is based on the Regge formalism [7] and leads to the formulation known as dynamical triangulations [8-12]: space-time configurations (triangulations) are represented by the possible collections of flat simplexes, glued together so as to reproduce different possible geometries. In the particular approach known as causal dynamical triangulations (CDT) [13-19], the causal condition of global hyperbolicity [20,21] is additionally enforced on triangulations by means of a space-time foliation, with spatial slices characterized by a fixed topology (usually $S^{3}$ ), and typically periodic boundary conditions (p.b.c.) in the time direction.

The path integral over triangulations is built by discretizing the candidate continuum action. In the absence of matter fields, the simplest candidate is the Regge discretized version of the Einstein-Hilbert action, which, after uniformly fixing the lattice spacings and performing a Wick rotation to Euclidean space (see Ref. [13] for more details), takes the form

$$
S_{E}=-k_{0} N_{0}+k_{4} N_{4}+\Delta\left(N_{4}+N_{41}-6 N_{0}\right),
$$

where $N_{0}, N_{4}$ and $N_{41}$ count, respectively, the total number of vertices, of generic pentachorons and of special pentachorons having four vertices on the same spatial slice, while $k_{4}, k_{0}$ and $\Delta$ are free dimensionless parameters, related to the cosmological and Newton constants $\Lambda$ and $G$, and to the time/space asymmetry of the building simplexes; such a correspondence is of course just at the level of the naive continuum limit since the connection with physical 
renormalized constants can be fixed only by the analysis around the actual continuum limit, if any is found. Triangulations are then sampled according to a distribution $\propto e^{-S_{E}}$, with the caveat that $k_{4}$ is usually traded for a target volume $V$, by adding to $S_{E}$ a volume fixing term: such a term does not change the original path integral weight among triangulations sharing the same volume.

A rich phase structure has been found for CDT $[13,15,17,18,22]$, characterized by four different phases, named $A, B, C_{d S}$ (de Sitter) and $C_{b}$ (bifurcation). In the $B$ phase, $V_{S}$ is concentrated almost in a single slice, while both the $C_{d S}$ and the $C_{b}$ phase are characterized by a more regular spatial volume distribution, localized in a so-called "blob" of finite time extension; finally, phase $A$ configurations are characterized by multiple and uncorrelated peaks in the spatial volume per slice $V_{S}(t)$. The bifurcation phase is further differentiated from the $C_{d S}$ phase by the presence of two different classes of slices which alternate with each other in Euclidean time [17,18,23]. The transition lines among those phases are the candidate places where one can search for a continuum limit, if they are second order: a candidate transition, in this respect, is the one separating the $C_{b}$ from the $C_{d S}$ phase. Moreover, $C_{d S}$ is the candidate phase for a correspondence with continuum QG, showing an effective dimension four at large scales and a volume distribution compatible with a de Sitter universe [13].

One of the major problems of the CDT program is to find suitable observables, capable of capturing the essential geometrical features around the transition. Progress has been achieved by the study of diffusion processes on the triangulations [24,25], leading to relevant information such as their spectral dimension. A generalization along this direction has been proposed in Ref. [26], consisting in the analysis of the spectrum of the Laplace-Beltrami (LB) operator computed on the triangulations.

The analysis of Ref. [26], limited to the LB operator defined on spatial slices, has shown that the various phases can be characterized by the presence $(B)$ or absence $\left(A, C_{d S}\right)$ of a gap in the spectrum, while the $C_{b}$ phase shows the alternance of spatial slices of both types, gapped and nongapped, which for this reason can be named $B$-type and $d S$-type slices. The presence of a gap indicates that spatial slices are characterized by a high connectivity and can be interpreted geometrically as a universe with an infinite dimensionality at large scales, whose diameter grows at most logarithmically with $V_{S}$. On the contrary, the closing of the gap indicates the emergence of an extended universe, with a finite dimensionality at large scales. In the $C_{b}$ phase, the alternating slices share similar geometries up to some finite length, then differentiate at larger scales. Moreover, the value of the gap seems to change continuously, moving from the $B$ to the $C_{b}$ phase, approaching zero towards the $C_{d S}$ phase.

The findings reported above, and the fact that the gap of the LB operator spectrum is a quantity with mass dimension two (an inverse squared length), suggest that the gap can be used as an order parameter for the $C_{b}-C_{d S}$ transition and, if it is second order, to characterize the critical behavior around it. Having this in mind, the purpose of this study is to put the strategy of Ref. [26] on firmer and more quantitative grounds. One should first demonstrate that the gap is actually a good order parameter by studying its thermodynamical limit, proving it is strictly zero in one phase and nonzero in the other. Once this is done, one can study its possible critical behavior around the transition: we see that one can actually find several length scales, all showing a similar scaling.

\section{NUMERICAL SETUP}

We have investigated CDT with p.b.c. in the Euclidean time direction and an $S^{3}$ topology for spatial slices. Configurations have been sampled proportionally to $\exp \left(-S_{E}\right)$ by means of a Metropolis-Hastings algorithm, consisting in a set of local moves (see Ref. [13] for more details). Here $N_{t}=80$ total space-time slices have been taken in all simulations, and the total spatial volume $V_{S \text {,tot }}=N_{41} / 2$ has been fixed by adding to $S_{E}$ a term $\Delta S=\epsilon\left(N_{41}-\bar{N}_{41}\right)^{2}$, with $\epsilon=0.005$, then selecting only configurations with $N_{41}=\bar{N}_{41}$.

The eigenvalues of the discretized LB operator have been computed on the spatial slices of those configurations, consisting of sets of glued tetrahedra. As in Ref. [26], the discretization consists of a linear operator $L$ acting on real functions defined on the vertices of the graph dual to the triangulation. Since any tetrahedron is adjacent to exactly 4 neighboring tetrahedra, dual graphs are 4-regular (each vertex is connected with 4 other vertices), so $L$ can be written as $L=4 \cdot \mathbb{1}-A$ where $A$ is the so-called adjacency matrix, having nonzero unit elements only between pairs of connected vertices. Therefore, $L$ is a sparse matrix, and its eignvalues have been computed by means of the "Armadillo" C++ library [27] with Lapack, Arpack and SuperLU support.

The smallest eigenvalue, $\lambda_{0}=0$, always corresponds to a uniform eigenfunction. Then, $\lambda_{1}$ defines the gap of the spectrum. We will study $\lambda_{1}$ and a few other lowest lying eigenvalues as a function of the spatial volume $V_{S}$, trying to extrapolate the $V_{S} \rightarrow \infty$ (thermodynamical) limit for each of them. For a regular, extended geometry one expects $\lambda_{n} \rightarrow 0$ in the thermodynamical limit for any finite $n$, in particular, $\lambda_{n} \propto 1 / D^{2}$ where $D$ is the diameter of the graph (maximum over all pairs of vertices of the minimum path length connecting the pair).

We have performed sets of simulations at fixed $k_{0}$ and different values of $\Delta$, chosen so as to stay in the $C_{b}$ phase while approaching the $C_{d S}$ phase; moreover, different values of $\bar{N}_{41}$ have been considered to explore the impact on results of the total spatial volume. A few simulations in the $B$ phase or in the $C_{d S}$ phase have also been performed to 
have a comparison for the infinite volume behavior of the lowest lying eigenvalues in those cases.

\section{RESULTS}

We start by analyzing the $V_{S} \rightarrow \infty$ limit of the lowest lying part of the LB spectrum in the $C_{d S}$ phase. We have considered a simulation performed for $k_{0}=0.75, \Delta=0.7$, with the total spatial volume fixed to $V_{S \text {,tot }}=410^{4}(40 \mathrm{~K})$. Since for each configuration most of the spatial volume is distributed over many connected slices forming the socalled blob, the volume $V_{S}$ of a single spatial slice is regularly distributed over a wide range going up to a few thousand tetrahedra. Because of our finite sample, consisting of about 500 configurations, this range has been divided into regular bins of volumes, so as to have sufficiently populated subsamples in each bin. Average values $\left\langle\lambda_{n}\right\rangle$ have then been computed over each bin: results for $n=1$, 3, 5 are reported in Fig. 1; statistical errors have been computed by properly taking into account autocorrelations among subsequent triangulations. For a few bins, we also report results obtained by fixing a different global volume $V_{S, \text { tot }}$, to check that this has no impact on the study of the $V_{S} \rightarrow \infty$ limit.

In Fig. 1 we also report best fits to a power law dictated by a large distance effective dimension $d_{E F F}$,

$$
\left\langle\lambda_{n}\right\rangle=A_{n} V_{S}^{-2 / d_{E F F}}
$$

all yielding $\chi^{2} /$ d.o.f. $\sim 1$ with $d_{E F F} \simeq 1.6$, in agreement with the large scale spectral effective dimension of spatial slices measured in previous studies [26,28]; similar results are obtained for $n$ up to a few tens. This confirms that, in the $C_{d S}$ phase, the gap of the LB operator closes in the thermodynamical limit, with a scaling dictated by $d_{E F F}$.



FIG. 1. Average eigenvalues of the LB operator on spatial slices versus $1 / V_{S}$ in phase $C_{d s}\left(k_{0}=0.75, \Delta=0.7\right)$, mostly for $V_{S \text {,tot }}=40 \mathrm{~K}$. We also report best fits to Eq. (2).
The situation is quite different in phase $B$. In this case, most of the total spatial volume is found in a single slice, so that, in order to study the $V_{S} \rightarrow \infty$ limit of the spectrum, we had to perform simulations at different values of $V_{S \text {,tot }}=3 \mathrm{~K}, 4 \mathrm{~K}, 5 \mathrm{~K}, 6.5 \mathrm{~K}, 8 \mathrm{~K}$. Results for $\left\langle\lambda_{n}\right\rangle$ on this single slice are reported, for some $n$, in Fig. $2 ; V_{S}$ in this case is the average volume of the maximal slice; statistical errors are reported but are not appreciable. In this case, a smooth infinite volume extrapolation is obtained by considering simple power corrections in $V_{S}^{-1}$ :

$$
\left\langle\lambda_{n}\right\rangle_{V_{S}}=\left\langle\lambda_{n}\right\rangle_{\infty}+\frac{a_{n}}{V_{S}}+\frac{b_{n}}{V_{S}^{2}}
$$

and $\chi^{2} /$ d.o.f. $\simeq 1$ is obtained only allowing for $b_{n} \neq 0$. As already expected from the results of Ref. [26], the extrapolated values $\left\langle\lambda_{n}\right\rangle_{\infty}$ are nonzero, as shown in Fig. 2. What is more interesting is that the extrapolated values for different values of $n$ do not coincide; i.e., in the thermodynamical limit the spectrum above the gap seems to be discrete, defining a hierarchy of length scales for the geometry of the $B$ phase. Further evidence comes from the behavior of the volume-normalized spectral density, which becomes smaller and smaller as $V_{S} \rightarrow \infty$ in the region above the gap, as expected for a discrete spectrum.

Our interest is mostly focused on the intermediate phase $C_{b}$. As discussed above, the two classes of alternating slices differ mostly at large scales. This is enlightened by the scaling profiles where the eigenvalues $\lambda_{n}$ of the LB operator are plotted versus the scaling variable $n / V_{S}$ : such profiles provide information about the effective dimensionality of spatial triangulations at different scales [26] (smaller $n / V_{S}$ corresponding to larger scales), $2 / d_{E F F}=d \log \lambda_{n} / d \log \left(n / V_{S}\right)$. In particular, the development of a gap in the $V_{S} \rightarrow \infty$ limit corresponds to an infinite dimensionality at large scales, induced by a high

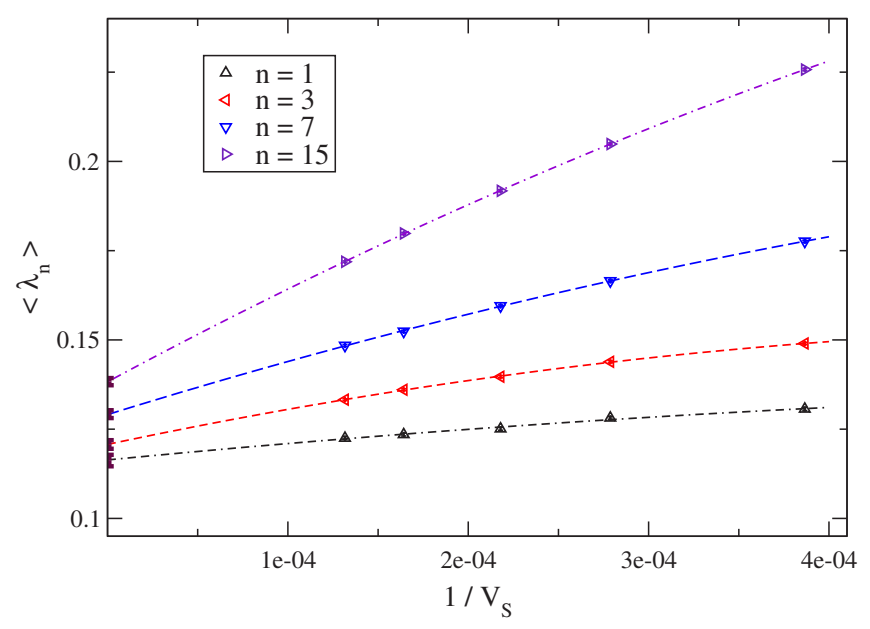

FIG. 2. Average eigenvalues versus $1 / V_{S}$ in phase $B\left(k_{0}=1.0\right.$, $\Delta=-0.2$ ). Data points at $1 / V_{S}=0$ have been extrapolated according to Eq. (3). 


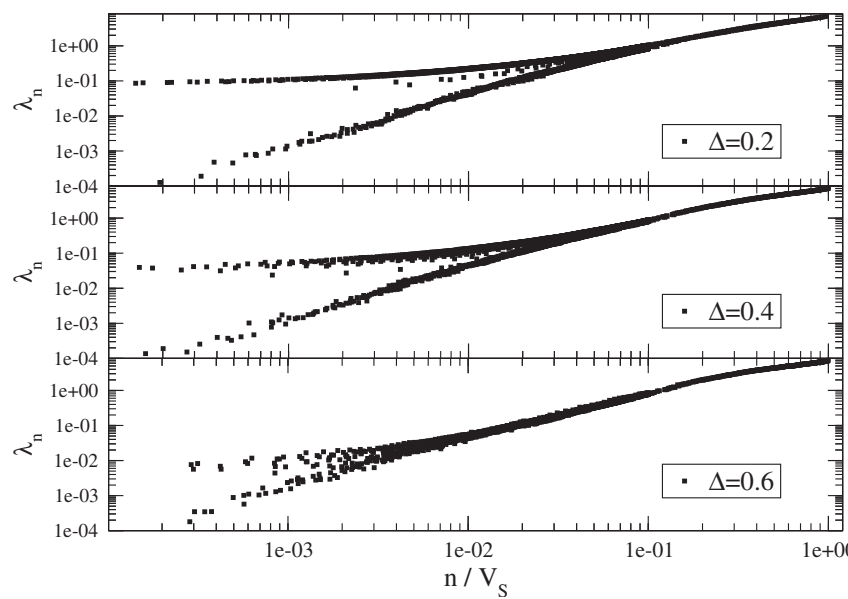

FIG. 3. Scatter plot of $\lambda_{n}$ versus $n / V_{S}$ for slices with $V_{S}>200$ from sample configurations at $k_{0}=0.75$ and three values of $\Delta$ in the $C_{b}$ phase.

connectivity of the dual graph. In Fig. 3 we report the profiles obtained in the $C_{b}$ phase at $k_{0}=0.75$ for three different values of $\Delta=0.2,0.4$ and 0.6 : the point where two different profiles emerge moves to smaller values of $n / V_{S}$ as $\Delta$ approaches the transition to the $C_{d S}$ phase, where the separation in two classes disappears. We have computed $\left\langle\lambda_{n}\right\rangle\left(V_{S}\right)$ separately for the two classes of slices, starting from $V_{S}$ large enough to make the separation unambiguous as in Fig. 3. Figure 4 shows an example of results obtained for $k_{0}=0.75$ and $\Delta=0.4$ on $d S$-like slices: an extrapolation as in Eq. (2) works well in all cases, with $\chi^{2} /$ d.o.f. $\simeq 1$, confirming the absence of a gap.

Results for $B$-like slices, reported for the same parameters in Fig. 5, point instead clearly to $\left\langle\lambda_{n}\right\rangle_{\infty} \neq 0$ in the $V_{S} \rightarrow \infty$ limit. Extrapolated values in Fig. 5 are the result of a fit to Eq. (3) and include systematic errors related to the choice of the fitted range or the inclusion/exclusion of $1 / V_{S}^{2}$ corrections. Even taking these systematics into



FIG. 4. $\left\langle\lambda_{n}\right\rangle\left(V_{S}\right)$ for $d S$-like slices in the $C_{b}$ phase $\left(k_{0}=0.75\right.$, $\left.\Delta=0.4, V_{S, \text { tot }}=40 \mathrm{~K}\right)$. We report also best fits to Eq. (2).



FIG. 5. As in Fig. 4, for $B$-like slices. We report also some $V_{S} \rightarrow \infty$ extrapolations with $1 / V_{S}$ corrections (see text).

account, $\left\langle\lambda_{n}\right\rangle_{\infty}$ values for different $n$ are not compatible, confirming that also for $B$-like slices the lowest lying part of the spectrum is likely discrete even in the thermodynamical limit, as for the $B$ phase.

The main point of our investigation is to adopt the nonzero $\left\langle\lambda_{n}\right\rangle_{\infty}$ of $B$-like slices as order parameters approaching zero at the $C_{b}-C_{d S}$ transition and probing a possible critical behavior around there. To that purpose, we performed simulations along two different lines with fixed $k_{0}$ ( $k_{0}=0.75$ and $k_{0}=1.5$ ), crossing the $C_{b}-C_{d S}$ transition line in different points, as sketched in Fig. 6. In Fig. 7 we report some $\left\langle\lambda_{n}\right\rangle_{\infty}(n=1$ and 5) as a function of $\Delta$ along these two lines; volumes where $B$-like and $d S$-like slices are clearly distinguished grow approaching the $C_{d S}$ phase, so that for some values of $\Delta$, which are excluded from Fig. 7, we could not reliably perform the $V_{S} \rightarrow \infty$ limit.

On dimensional grounds, different $\left\langle\lambda_{n}\right\rangle_{\infty}$ correspond to different inverse squared lengths, which around a critical

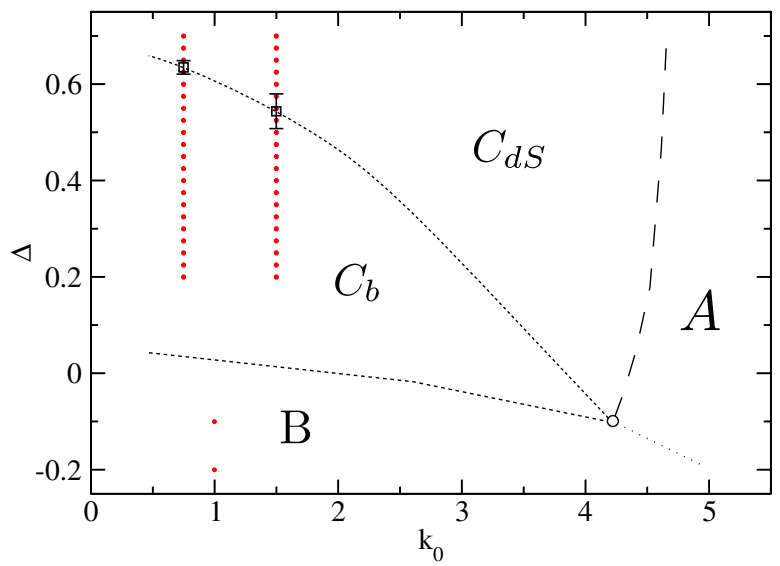

FIG. 6. Sketch of the phase diagram of CDT with $S^{3}$ slice topology. The isolated points correspond to the simulations performed (total volume not shown); The points with error bars are the quantitative estimates for the corresponding critical values of $\Delta_{c}$ obtained in this study and reported in the text. 


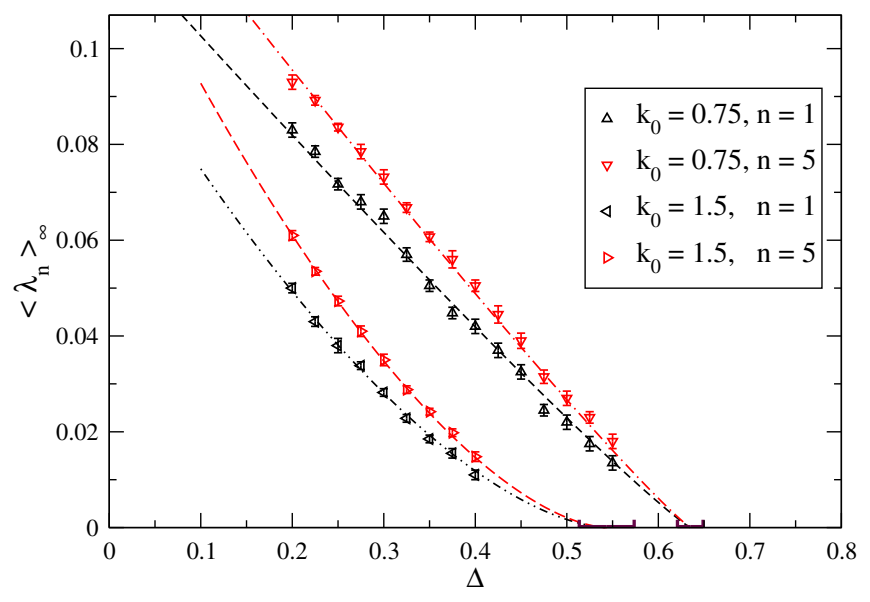

FIG. 7. $\left\langle\lambda_{n}\right\rangle_{\infty}$ in $B$-like slices as a function of $\Delta$ for different values of $k_{0}$ and $n$, together with best fits to Eq. (4).

point should scale proportionally to each other. Based on that, we have tried a fit to the scaling formula

$$
\left\langle\lambda_{n}\right\rangle_{\infty}=A_{n}\left(\Delta_{c}-\Delta\right)^{2 \nu}
$$

where only the $A_{n}$ coefficients depend on $n$. A combined fit, including $n=1,5$, yields $\Delta_{c}=0.635(14), \nu=0.55(4)$ for $k_{0}=0.75\left(\chi^{2} /\right.$ d.o.f. $\left.=31 / 26\right)$, and $\Delta_{c}=0.544(36)$, $\nu=0.82(12)$ for $k_{0}=1.50\left(\chi^{2} /\right.$ d.o.f. $\left.=6 / 14\right)$. Similar and consistent results are obtained including different values of $n$, or if the eigenvalues are fitted separately; some tension emerges only when eigenvalues with $n \gtrsim 10$ are included. Our best fits suggest that the index $\nu$ could change along the transition line; however, we stress that a global fit in which $\nu$ is set equal for both $k_{0}$ works equally well, yielding $\nu=0.59(4), \Delta_{c}\left(k_{0}=0.75\right)=0.656(15)$, $\Delta_{c}\left(k_{0}=1.5\right)=0.479(10)$ with $\chi^{2} /$ d.o.f. $=47 / 41$.

\section{CONCLUSIONS}

We have studied the spectrum of the LB operator defined on the foliation slices of CDT, showing that the $B$ and the
$C_{b}$ phases of the theory are characterized by a discrete set of lowest eigenvalues, persisting in the thermodynamical limit. They can be interpreted as a set of dynamical mass scales, characterizing, for instance, the propagation of massless scalar fields in the sampled geometries.

When the appropriate infinite volume limit is taken, these mass scales appear to vanish as the de Sitter phase is approached, thus permitting us to determine the location of the transition from the $C_{b}$ to the $C_{d s}$ phase. It is interesting to stress that such a determination, which is reported in Fig. 6 for the two explored values of $k_{0}$, is compatible with what can be found by the analysis of other parameters, based on counting the coordination number of triangulations, which have been introduced and used in previous studies [18,29].

The novelty of the quantities analyzed in this study is that they vanish exactly in one phase, as one would expect for an order parameter. Moreover, these scales have a dependence on the bare couplings which is consistent with a common critical behavior, i.e., governed by a common critical exponent: this is exactly what one would expect for a lattice field theory approaching the continuum limit.

We stress that such length scales are still not of direct physical relevance. However, our findings create a clear path for future steps along the CDT program. If analogous length scales are defined on the other side of the transition, in the $C_{d S}$ phase, one could then check if they scale as a function of the bare parameters, similarly to what was found in this study: that would provide clear evidence for the existence of a critical point.

\section{ACKNOWLEDGMENTS}

Numerical simulations have been performed on the MARCONI machine at CINECA, based on the agreement between INFN and CINECA (under Project No. INF18_npqcd) and at the IT Center of the Pisa University. We thank, in particular, M. Davini for his technical support.
[1] M. H. Goroff and A. Sagnotti, Nucl. Phys. B266, 709 (1986).

[2] S. Weinberg, General Relativity, an Einstein Centenary Survey (Cambridge University Press, Cambridge, England, 1979), Chap. 16.

[3] D. F. Litim, Phil. Trans. R. Soc. A 369, 2759 (2011).

[4] M. Reuter, Phys. Rev. D 57, 971 (1998).

[5] M. Reuter and F. Saueressig, New J. Phys. 14, 055022 (2012).

[6] A. Bonanno and M. Reuter, Phys. Rev. D 65, 043508 (2002).

[7] T. Regge, Nuovo Cimento 19, 558 (1961).
[8] J. Ambjorn and J. Jurkiewicz, Phys. Lett. B 278, 42 (1992).

[9] M. E. Agishtein and A. A. Migdal, Mod. Phys. Lett. A 07, 1039 (1992).

[10] J. Ambjorn, J. Jurkiewicz, and C. F. Kristjansen, Nucl. Phys. B393, 601 (1993).

[11] T. Rindlisbacher and P. de Forcrand, J. High Energy Phys. 05 (2015) 138.

[12] J. Laiho, S. Bassler, D. Coumbe, D. Du, and J. T. Neelakanta, Phys. Rev. D 96, 064015 (2017).

[13] J. Ambjorn, A. Goerlich, J. Jurkiewicz, and R. Loll, Phys. Rep. 519, 127 (2012). 
[14] J. Ambjorn, J. Jurkiewicz, and R. Loll, Phys. Rev. Lett. 85, 924 (2000).

[15] J. Ambjorn, S. Jordan, J. Jurkiewicz, and R. Loll, Phys. Rev. Lett. 107, 211303 (2011).

[16] J. Ambjorn, S. Jordan, J. Jurkiewicz, and R. Loll, Phys. Rev. D 85, 124044 (2012).

[17] J. Ambjorn, J. Gizbert-Studnicki, A. Goerlich, J. Jurkiewicz, N. Klitgaard, and R. Loll, Eur. Phys. J. C 77, 152 (2017).

[18] J. Ambjorn, D. Coumbe, J. Gizbert-Studnicki, A. Goerlich, and J. Jurkiewicz, Phys. Rev. D 95, 124029 (2017).

[19] J. Ambjorn, J. Gizbert-Studnicki, A. Goerlich, K. Grosvenor, and J. Jurkiewicz, Nucl. Phys. B922, 226 (2017).

[20] E. Minguzzi and M. Sanchez, Recent Developments in Pseudo-Riemannian Geometry, edited by $\mathrm{H}$. Baum and D. Alekseevsky (EMS Pub. House, Zurich, 2008), pp. 299-358.
[21] S. Jordan and R. Loll, Phys. Rev. D 88, 044055 (2013).

[22] J. Ambjorn, J. Gizbert-Studnicki, A. Goerlich, J. Jurkiewicz, and D. Nemeth, J. High Energy Phys. 06 (2018) 111.

[23] J. Ambjorn, J. Gizbert-Studnicki, A. Goerlich, and J. Jurkiewicz, J. High Energy Phys. 06 (2014) 034.

[24] D. N. Coumbe and J. Jurkiewicz, J. High Energy Phys. 03 (2015) 151.

[25] J. Ambjorn, J. Jurkiewicz, and R. Loll, Phys. Rev. Lett. 95, 171301 (2005).

[26] G. Clemente and M. D’Elia, Phys. Rev. D 97, 124022 (2018).

[27] C. Sanderson and R. Curtin, J. Open Source Software 1, 26 (2016).

[28] A. Goerlich, arXiv:1111.6938.

[29] J. Ambjorn, D. N. Coumbe, J. Gizbert-Studnicki, and J. Jurkiewicz, J. High Energy Phys. 08 (2015) 033. 